\title{
FPGA IMPLEMENTATION OF WIRELESS DATA LOGGER SYSTEM
}

\author{
K Mahananda ${ }^{1}$ \\ ${ }^{1} P G$ student, VTU Regional office, Kusnoor road Kalaburagi
}

\begin{abstract}
This Project describes the design and development of hardware and software modules for wireless data logger system using ZigBee.. FPGA firmware is developed in VHDL using Xilinx ISE 14.7 and the design is simulated using ModelSim Simulator and the code is synthesized on Altera Cyclone IV FPGA.End user can change remotely the data logger settings such as date and time. The Advent of Field Programmable Gate Arrays (FPGAs) has made compact realization of embedded system possible. As FPGAs are high density logic devices. It is possible to realize certain software functions in hardware in an efficient way.
\end{abstract}

Keywords: Data logger; FPGA; ZigBee; Real time clock; ADC.

****

\section{INTRODUCTION}

The main aim of the project is to design and implementation of an wireless data logger on FPGA, which will accept physical parameters like temperature, humidity etc from the sensor and is converted into digital data by ADC and then stored in the memory that is in different latches that data can be used according to the different application. With emerging technology, researchers desire to obtain their sensor data faster and to make accepted data available over longer interval of time.The primary transformative trend of data loggers has been in size reduction. This is because of their primary role of remote data redemption. They need to be haulable to facilitate easy portage.

Traditional general purpose processors [GPP] and wired communication devices requires high cost and long development cycle. As the GPP have the fixed architecture, limitation to data path width, so it may waste the hardware resources. FPGA gives the flexibility of reprogramming and have high level parallelism. Wired communication possess several limitations like complex cable connections, electromagnetic interference, wire cost and maintenance etc. There are several wireless communication technologies, such as Bluetooth, WSN, WI-FI, ZigBee, IrDA, GSM etc. Taking in to account the application range of various techniques, the cost of data transmission, security and reliability, Bluetooth and WSN are the most appropriate methods in the short range wireless data communication and data transmission. ZigBee is a low cost, low power built from small digital radios targeted at wide development of long battery life devices in wireless control and monitoring applications.

The mobile communication devices are mostly focused on voice and short messaging. They have become one of the inseparable parts of the people life for contacting each other via text and voice and it also provides services like check mail, play game, send and receive file, etc. The combination of these two services is called as smart phone. The Android OS is the fastest growing OS on the mobile market. Furthermore, it is open source; thus allowing more ease and freedom when developing applications. One of the primary benefits of using data loggers is the ability to automatically collect data on a 24-hour basis, and time stamp of logging can be set (five minutes to one hour basis). Upon activation, data logger is left unattended to measure and record information for the duration of the monitoring period.

\section{DESIGN METHODOLOGY}

Below Figure 2.1 shows the basic block diagram of Wireless data logger system, which divides the figure into two main parts one is called as Transmitter block system and the other is Receiver block system. The common wireless data logger system is Full Duplex communication.

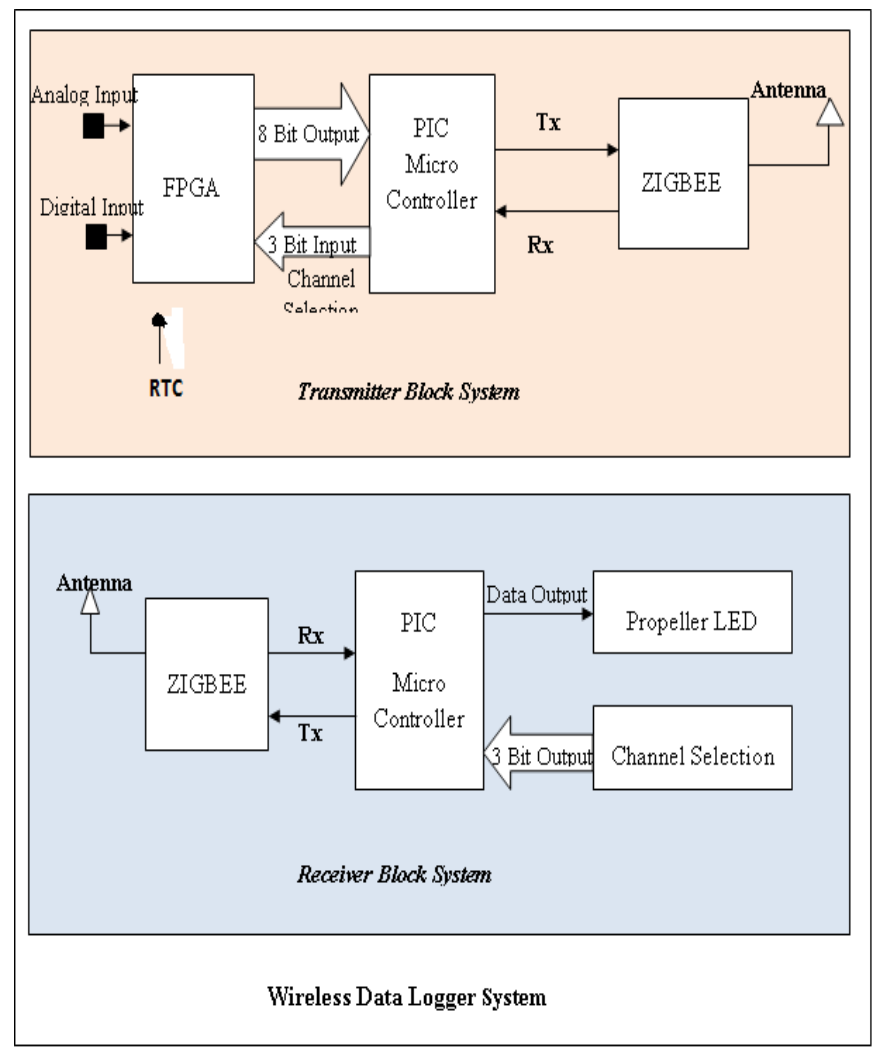

Fig 2.1: Basic Block Diagram of Wireless Data Logger System 


\subsection{Transmitter Block System}

In above figure 2.1 transmitter block consist of FPGA, PIC Microcontroller, ZigBee and Real time clock. In this block, information is transferred in both directions at a time so it is a "Full Duplex Communication". Now we will see how the data is transferred in each block. FPGA takes Data from the sensor as well as from the microcontroller as the communication is a full duplex. First FPGA takes a data from the sensor as input which stores data continuously in the different channel memory block, as FPGA contains total 8 channel memory block these blocks are divided with respect to time slots allocated for the channel. Second FPGA takes 3bit channel address from the microcontroller, then the required data which is stored in the particular channel memory block the data is sent back to the micro controller which as an 8bit data. The switching action is controlled by the PIC microcontroller, the controls of these switching actions are programmed into memory and actions are executed by the FPGA. So this block is called as "Stored program control switching system".

Microcontroller takes channel address from the ZigBee and then decodes that channel address into three bit. The decoded channel address is sent to an FPGA by the Microcontroller. Then whatever the data which is of 8 bit stored in that channel address is sent back to the Microcontroller by the
FPGA, as FPGA stores data in different memory block that is in different eight Latches. Now Microcontroller encodes this 8 bit data and transfers this data over the ZigBee. By implementing ZigBee protocol the communication between the hardware and the software unit of the system is made wireless easily so that the ZigBee takes DATA IN as channel address and DATA OUT as data for the selected channel.

\subsection{Receiver Block System}

The Receiver Block System also consists of three main blocks ZigBee, PIC Microcontroller, and propeller LED as show in the above figure 2.1.Now we will see how the data transfer take place in this block as here the communication is half duplex. The microcontroller sends channel address to the ZigBee and by the wireless transmission it takes data from the transmitter as explained in the transmitter block system, as here the communication is a half duplex. And that data from the ZigBee is decoded and displayed on the propeller LED.

The operation of ZigBee is controlled by the microcontroller by switching program. here First ZigBee Sends DATA OUT as this data contains channel address and takes DATA IN as this data contains data for the selected channel.

\section{SIMULATION RESULTS}

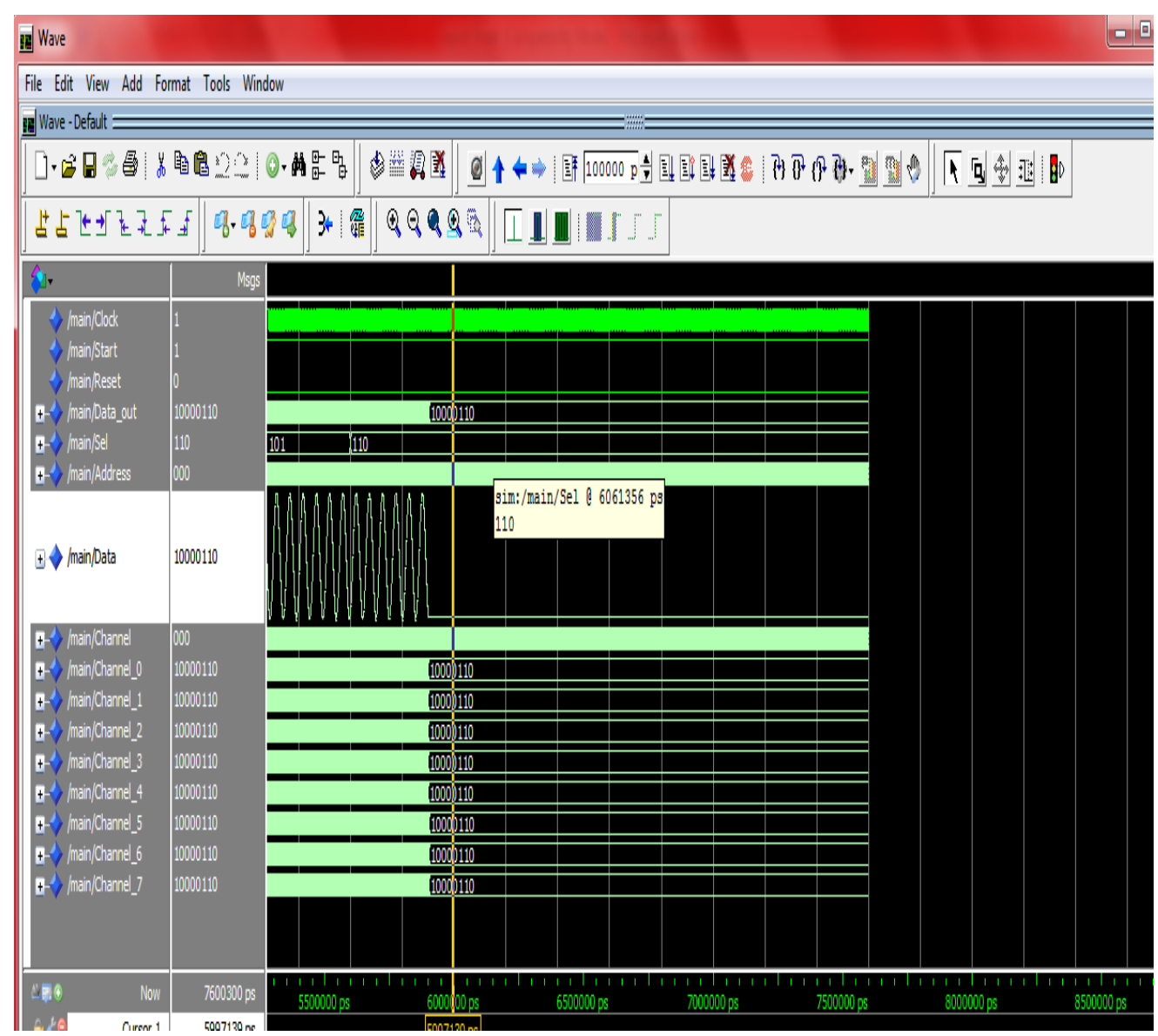

Fig 3.1 Simulation result of data logger 
Above fig 3.1 describes simulation results of Data logger. First give RESET as HIGH, START as HIGH and give CLK, now output is 00000000.Then make RESET as LOW and now we are assuming here continuous varying temperature shows as analog signal, for each 1 second the data will be stored in each channel, then after every 8 second the data will be updated in each channel. Suppose now if we are selected particular channel say channel address 110, whatever the data in sixth channel presently that will be the Data_out.

Table 1: Design Summary

\begin{tabular}{|l|l|l|l|}
\hline \multicolumn{2}{|l|}{ Device utilization values(estimated values) } \\
\hline $\begin{array}{l}\text { Logic } \\
\text { utilization }\end{array}$ & Used & $\begin{array}{l}\text { Availabl } \\
\text { e }\end{array}$ & $\begin{array}{l}\text { Utiliz } \\
\text { ation }\end{array}$ \\
\hline $\begin{array}{l}\text { Number of } \\
\text { slices }\end{array}$ & 46 & 960 & $4 \%$ \\
\hline $\begin{array}{l}\text { Number of slice } \\
\text { flip flops }\end{array}$ & 14 & 1920 & $0 \%$ \\
\hline $\begin{array}{l}\text { Number of 4 } \\
\text { input LUTs }\end{array}$ & 86 & 1920 & $4 \%$ \\
\hline $\begin{array}{l}\text { Number of } \\
\text { bonded IOBs }\end{array}$ & 11 & 66 & $16 \%$ \\
\hline $\begin{array}{l}\text { Number of } \\
\text { GCLKS }\end{array}$ & 1 & 24 & $4 \%$ \\
\hline
\end{tabular}

\section{CONCLUSION AND FUTURE SCOPE}

This Project mainly deals with the Data logger system which plays an important role in diverse application such as chemical plants, process control industries, and satellites. An data logger system interacts continuously with its environment and carries out various tasks with certain timing constraints. The Data logger which has been implemented in hardware for redemption process and this reduces the software overhead and hence the execution cycle time that makes the system response faster in real-time. This leads to a better performance and accuracy.

All the functional modules of Data Logger all functional blocks are successfully coded in VHDL, simulated and verified as per the functional requirement and timing specification and hardware implementation is done on the FPGA.

The FPGA based wireless data logger presented in this project records temperature and humidity data over a period of time. It displays stored data on propeller LED when we selected particular channel address. This data can be used for future analysis of the environmental conditions at the place where data logger is installed.

\section{REFERENCES}

[1] C. A. Gómez-Pachón, M. Valderrama, F. SeguraQuijano, "Design and Implementation of a Wireless Tensiometer with Smartphone Interface" Health Care Exchanges (PAHCE), 2013 Pan American, pp. 1-3, April-May 2013.
[6] "Preliminary Design document of Data Acquisition and Command processing," January 2008, Control System Group, ISRO Satellite Centre.

[7] Swamy TN, Rashmi KM "Data Acquisition System Based On FPGA'-International Journal of Engineering Research and Applications

[8] Engelberg, S. ; Kaminsky, T. ; Horesh, M. “A USBEnabled, FLASH-Disk-Based Data Logger'Instrumentation \& Measurement Magazine, IEEE (Volume:10, Issue: 2 ) April 2007

[2] Gopinath Shanmuga Sundaram, Bhanu Prasad Patibandala, "Bluetooth Communication using a Touch screen Interface with Raspberry Pi," Southeastcon, 2013 Proceedings of IEEE,pp. 1-4, April 2013. BouSaba, "Real time Bluetooth Communication Between an FPGA Based Embedded System and an IEEE, pp. 1-4, April 2013.

LetianXu ; Ocean Coll., Zhejiang Univ., Hangzhou, China ; Jiawang Chen ; Ying Chen ; Xiaoping Hu "A San Diego, 2013

Wei Siew; "Design and development of a High Tech Congress on Electronics (GHTCE), 2012 IEEE 18-20 Nov. 2012

9] FPGA Implementation of Real Time Data Acquisition System Using Micro blaze ProcessorD.SathishkumarR.Ganesh International Journal of Science and Engineering Applications Volume 2 Issue 7, 2013, ISSN-2319-7560 (Online)

[10] Nkom, B. ; Eng. \&Instrum. Design Sect., "Development of a Novel Microcontroller-based data logger" Adaptive Science \& Technology, 2009. ICAST 2009. 2nd International Conference on 14-16 Jan. 2009
[3] SamerHawayek, Claude Hargrove and Nabila A. 